\title{
Roberts syndrome: clinical and cytogenetic aspects
}

\author{
N P MANN, J FITZSIMMONS, E FITZSIMMONS, AND P COOKE \\ From the Clinical Genetic Service, City Hospital, Nottingham NG5 IPB
}

SUMMARY Roberts syndrome is reported in two sibs of consanguineous parents. Both infants had severe tetraphocomelia, facial clefting, and other serious malformations. In addition they were found to have an unusual cytogenetic abnormality with distortion of the normal sister chromatid relationship in many chromosomes.

Roberts syndrome is a rare condition, originally described in 1919 and reported in fewer than 30 children since that time. ${ }^{1}$ There have been several comprehensive reviews of the published cases $^{2}$ and recently Herrmann and Opitz ${ }^{3}$ compared Roberts syndrome with another similar condition, the SC phocomelia or pseudothalidomide syndrome. They concluded, at the time of their report, that there were insufficient grounds for suggesting that these were different entities although there are undoubted differences in severity and life expectancy. Both conditions, with their striking limb and facial abnormalities, have been reported in sibs and from families with parental consanguinity. They have therefore been considered to be of genetic origin and recessively inherited.

In 1973, Judge ${ }^{4}$ reported an unusual chromosomal abnormality in the pseudothalidomide syndrome and similar findings were subsequently reported in Roberts syndrome. ${ }^{5}$ In both conditions the abnormalities consisted of unusual sister chromatid separation, 'puffing' of the chromosomes at the centromere, and a characteristic 'tram-track' appearance of the sister chromatids. To date no similar findings have been reported in other malformations, and this might suggest that these two syndromes are indeed one and the same entity with variation in the clinical manifestations.

Our two cases demonstrate all the previously described physical characteristics of Roberts syndrome and there were additional findings noted at necropsy. Both showed the characteristic cytogenetic abnormalities.

\section{Case reports}

CASE 1

An 18-year-old Pakistani Muslim primigravida gave birth to a female infant of 38 weeks' gestation at

Received for publication 10 June 1981
Nottingham City Hospital on 9.1.79. She and her husband were first cousins and the couple's mothers were sisters. She had been in good health throughout the pregnancy but was consistently noted to have a fundal height below that expected for her dates. There was no history of drug ingestion other than oral iron and she had not taken clonidine at any time. $^{6}$ The infant's birthweight was $1.040 \mathrm{~kg}$, and although there were a few spontaneous gasps she died 17 minutes after delivery. On examination (fig 1) the following abnormalities were noted. (1) Gross intrauterine growth retardation. (2) Severe reduction deformities of all four limbs: tetraphocomelia. In addition the hands showed ectrodactyly with only a thumb and two fingers present. There was syndactyly of the $3 \mathrm{rd}$, 4th, and 5th toes of both feet. (3) Bilateral cleft lip and palate, a flattened nose without nostrils, and a prominent premaxilla. (4) Microcephaly, hypertelorism, a short neck, and primitive ears. (5) The clitoris was grossly enlarged and the genitalia ambiguous on

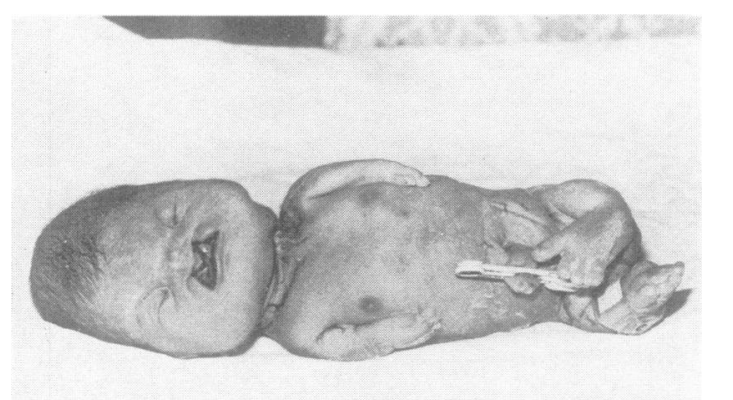

FIG 1 Infant showing typical features of Roberts syndrome (case 1). 
initial examination. The nipples were widely spaced and heavily pigmented.

On $x$-ray the vertebral bodies were noted to be flattened and there were short angled ribs. The femora were grossly shortened and it was difficult to recognise a tibia and fibula. At necropsy examination the kidneys were multicystic and dysplastic; both ureters were present. The lungs were grossly hypoplastic and there was no evidence of congenital heart disease. Both frontal lobes of the brain were small and there was absence of both olfactory lobes.

Four months after the birth of their first child the parents attended the genetic counselling clinic. Prenatal diagnosis was discussed but the parents did not favour termination of pregnancy and this type of help was refused. It was subsequently discovered that the wife was already 7 weeks pregnant at the time of her visit to the clinic.

\section{CASE 2}

The second affected infant, a male, was stillborn at 31 weeks' gestation weighing $500 \mathrm{~g}$. Abnormalities similar to, but more severe than, those in the first infant were present. The limbs were rudimentary with the hands consisting only of a thumb and two fingers. There was gross microcephaly and hypertelorism. The facial clefting was marked and in addition there was a frontal encephalocele. The ears were low-set and primitive. The genitalia were clearly male with an apparently enlarged phallus (fig 2).

$X$-ray examination confirmed defects in the long bones similar to those in case 1 . At necropsy, the frontal lobes were noted to be hypoplastic and the olfactory lobes absent. In addition, the left eye was microphthalmic and there was absence of the optic nerve on that side. There was a deficiency of the frontal bones with an encephalocele bulging through

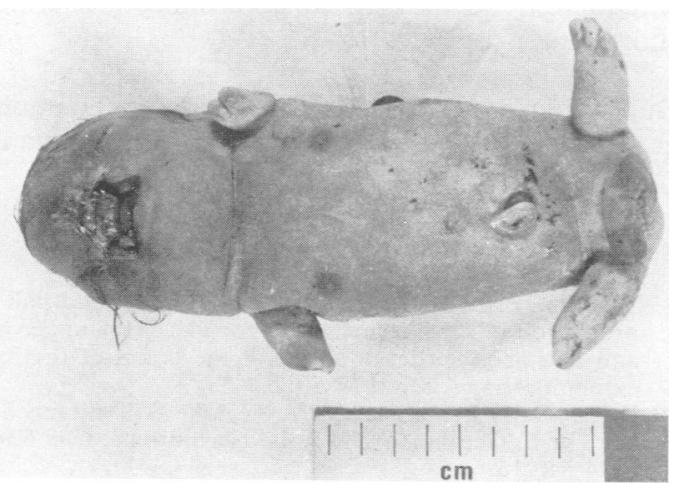

FIG 2 Case 2, more severely affected than case 1. There is apparent enlargement of the phallus. the gap. The lungs were grossly hypoplastic and incompatible with survival. The renal anomalies were severe with absence of the left kidney and ureter. The right kidney was dysplastic with multiple cysts. There was no evidence of oligohydramnios in either pregnancy, despite the renal dysplasia.

\section{CYTOGENETIC STUDIES}

Chromosome preparations from the first patient were obtained by microculture of heparinised blood $(0.5 \mathrm{ml}$ whole blood plus $0.2 \mathrm{ml}$ phytohaemmaglutinin in $10 \mathrm{ml}$ complete medium made up of a 3:1 mixture of TC199:fetal bovine serum). Harvesting took place after 72 hours following 6 hours of colcemid treatment $(0 \cdot 1 \mu \mathrm{g} / \mathrm{ml})$ and preparations were made using wet, ice cold, greasefree slides. Some were stained with Leishmann only and some were banded with trypsin. From the

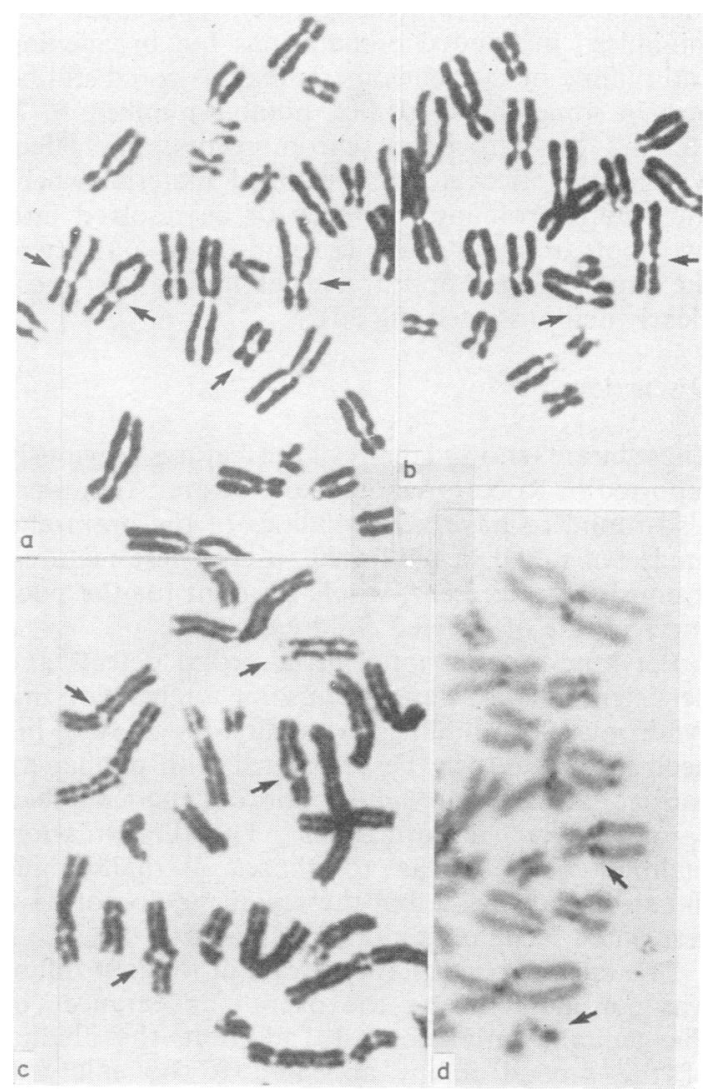

FIG $3(a, b)$ 'Tram-track' appearance of the chromosomes with puffing at the centromeres; (c) gross separation at centromeres; $(d) C$ banded preparations showing separated heterochromatic segments and widely splayed Y chromosome. 
second patient, fibroblast cultures were established from a post mortem skin biopsy and grown in a medium consisting of a 6:3:1 mixture of Ham's F10 medium, pooled human serum, and tryptone phosphate broth. Two sets of harvests were made, one after 3 hours of colcemid pretreatment and one after 6 hours. In addition to Leishmann staining and trypsin banding some material was $\mathrm{C}$ banded.

The cytogenetic abnormalities were present in cells from both patients (fig 3). Leishmann stained preparations showed separation of the chromatids at the proxicentric regions leading to the characteristic 'tram-track' appearance of some of the chromosomes (fig 3a, b). This sometimes gave the superficial appearance of chromosome breakage especially where acrocentric chromosomes were associated. The effect was most pronounced in early metaphase and so was seen most clearly in preparations from the second case which had been given relatively short colcemid treatment (fig 3c). The effect was minimised in banded preparations but broadening and puffing of the centromeric regions could still be seen in some chromosomes, notably numbers 9,7 , 16 , and the acrocentric chromosomes. The effect was most noticeable in $\mathrm{C}$ banded material where the heterochromatin was seen to be resolved into two spots in many of the chromosomes, and where the usual splayed appearance of the $\mathrm{Y}$ was most clearly demonstrated (fig 3d).

\section{Discussion}

These infants showed many of the features previously reported in Roberts syndrome. However, they were also found to have abnormalities of the brain and lungs not noted in published cases. These features, if consistently present, would account for the poor survival rate of affected children.

Previous reports noted atrial septal defects and persistent ductus arteriosus in some infants with this syndrome. Such findings in the first few days of life need not necessarily be associated with permanent cardiac defects and neither of our patients had cardiovascular abnormalities. The limb abnormalities were similar to those of thalidomide induced phocomelia, but these patients do not have associated facial or genital anomalies. ${ }^{7}$

The enlargement of the clitoris in the first infant was considerable and the overall appearance of the genitalia ambiguous. In addition, the phallus of the second infant appeared to be enlarged. Hintz et $a l^{8}$ have raised the possibility that the genital size of some infants may appear excessive because of their otherwise reduced body size. He further noted that surviving infants with Roberts or pseudothalidomide syndrome were not reported as having enlarged genitalia. Both of these observations could be explained in terms of different severity in the two conditions. Infants with Roberts syndrome usually die and their abnormalities are more severe than those of the pseudothalidomide syndrome. Our patients had abnormal genitalia with phallic enlargement and possibly increased nipple pigmentation. These could result from an associated endocrine disorder. The combination of facial clefts, absence of olfactory lobes, and small frontal lobes is similar to that seen in holoprosencephaly and this condition has been reported in association with endocrine dysgenesis. ${ }^{8}$

There does not appear to be any adequate explanation for the chromosomal changes seen in the condition. Freeman et $a l^{2}$ commented that the effect appeared to be independent of microtubule organisation in that it could be shown in cells both treated and untreated with colcemid. The characteristic splaying of the long arms of the $Y$ confirms that the phenomenon is not limited to the kinetochore but extends to all regions rich in $\mathrm{C}$ heterochromatin. The degree of distortion appears to be related to the amount of $C$ heterochromatin present and is most pronounced in chromosomes such as 1,9 , and 16 where the amount of $C$ banded material at the centromere is greatest. The parents in this case had normal karyotypes and there was no sign of splaying of the $\mathrm{Y}$ chromosome in the father. It appears likely that the effect is associated with altered behaviour rather than altered structure of the heterochromatic regions and is an expression, parallel to the clinical manifestations, of an underlying metabolic disturbance. Whatever its source, the chromosome picture is distinctive and detectable in fibroblasts. The condition should therefore be amenable to prenatal detection and amniocentesis should demonstrate the cytogenetic abnormalities. Ultrasonography in early pregnancy should also be of help in confirming gross limb shortening.

Our thanks are due to Mr G Gilbert for the photographs and Miss $\mathrm{K}$ Watson for typing the manuscript.

\section{References}

1 Roberts JB. A child with double cleft of lip and palate, protrusion of the intermaxillary portion of the upper jaw and an imperfect development of the bones of the four extremities. Ann Surg 1919;70:252-3.

2 Freeman MVR, Williams DW, Schimke N, Temtamy SA, Vachier E, German J. The Roberts syndrome. Clin Genet 1974;5:1-16.

${ }^{3}$ Herrmann J, Opitz JM. The SC phocomelia and Roberts syndrome: nosologic aspects. Eur J Pediatr 1977;125: 117-34. 
4 Judge C. A sibship with pseudothalidomide syndrome and an association with $\mathrm{Rh}$ incompatability. Med J Aust $1973 ; 2: 280-1$.

5 German J. Roberts' syndrome. 1. Cytological evidence for a disturbance in chromatid pairing. Clin Genet $1979 ; 16: 441-7$.

6 Stoll C, Levy JM, Beshara D. Roberts's syndrome and clonidine. J Med Genet 1979;16:486-8.

7 Fitzsimmons EM. Genetic counselling and Roberts syndrome. Nurs Mirror 1980;5:40-2.
${ }^{8}$ Hintz RLL, Menking M, Sotos JF. Familial holoprosencephaly with endocrine dysgenesis. Pediatr 1968;72: 81-7.

Requests for reprints to $\mathrm{Dr} \mathrm{J}$ S Fitzsimmons, Department of Clinical Genetics, City Hospital, Hucknall Road, Nottingham NG5 1PB. 